\title{
On the likelihood of Condorcet's profiles *
}

\author{
V. Merlin ${ }^{\dagger} \quad$ M. Tataru ${ }^{\ddagger} \quad$ F. Valognes ${ }^{\S}$
}

Revised version, june 2000

\begin{abstract}
Consider a group of individuals who have to collectively choose an outcome from a finite set of feasible alternatives. A scoring or positional rule is an aggregation procedure where each voter awards a given number of points, $w_{j}$, to the alternative she ranks in $j^{\text {th }}$ position in her preference ordering; the outcome chosen is then the alternative that receives the highest number of points. A Condorcet or majority winner is a candidate who obtains more votes than her opponents in any pairwise comparison. Condorcet [4] showed that all positional rules fail to satisfy the majority criterion. Furthermore, he supplied a famous example where all the positional rules select simultaneously the same winner while the majority rule picks another one. Let $P^{\star}$ be the probability of such events in three-candidate elections. We apply the techniques of Merlin, Tataru and Valognes [17] to evaluate $P^{\star}$ for a large population under the Impartial Culture condition. With these assumptions, such a paradox occurs in $1.808 \%$ of the cases.
\end{abstract}

\section{Introduction}

One of the simplest problems to state in collective decision theory is this. Consider a finite population $N=\{1,2, \ldots n\}$ together with a finite set of alternatives $A=\left\{a_{1}, a_{2}, \ldots a_{m}\right\}$. What method should society use in order to select an element of $A$ ? One natural method is to base social within decision on the preferences of individual society. Typically, each

\footnotetext{
* The authors are indebted to Ashley Piggins for his careful reading.

${ }^{\dagger}$ GEMMA-CREME and CNRS, MRSH-SH230, Université de Caen, Esplanade de la Paix, F-14032 Caen Cedex, France.

${ }^{\ddagger}$ Northwestern University, 2033 Sheridan Road, Evanston, IL 60208-2730 USA.

${ }^{\S}$ Department of Economics, The University of Namur, Rempart de la Vierge 8, B-5000 Namur, Belgium.
} 
individual is supposed to be rational, i.e., their preference relation is represented by a linear order on $A$. This basic framework captures many collective decision problems, from the selection of an investment project by a committee of experts, to the classical case of political elections.

Unfortunately, there is no unproblematic method for aggregating individual preferences in this setup. Two common principles are the 'positional' approach and the 'majority' method. Since the two-century old polemic between Borda and Condorcet, the supporters of the positional approach and the advocates of the majority principle have continued to provide new arguments in favor of their own views. More precisely, Borda [3] suggested that each voter should give $(m-1)$ points for the candidate she prefers, $(m-2)$ for her second best choice, and so on down to 1 point for the next to the last and 0 for her bottom ranked alternative. Then, the Borda score for the alternative $a_{k}$ is the number of points it receives over the whole population, and the Borda winner is the candidate whose Borda score is the highest. In fact, the Borda count belongs to a wider class of positional rules, also called scoring rules. A scoring rule is defined by a scoring vector $w=\left(w_{1}, w_{2}, \ldots w_{j}, \ldots w_{m}\right)$, $w_{1}>w_{m}, w_{j} \geq w_{j+1} \forall j=1, \ldots m-1$, where $w_{j}$ is the number of points an alternative receives each time it is ranked in $j^{\text {th }}$ position in one individual's preference. In this setup, the Borda count is naturally described by the scoring vector $w=(m-1, m-2, \ldots 2,1,0)$. The plurality rule, which selects as a winner the alternative with the highest number of first positions, is another famous scoring rule. It is defined by the vector $w=(1,0, \ldots 0)$. Finally, this class of decision processes also contains one of the veto methods: the antiplurality rule, in which each of the voters vetoes one alternative, and the candidate with the smallest opposition wins. It is equivalent to the positional rule described by the scoring vector $w=(1, \ldots 1,0)$.

In his speech before the Académie Royale des Sciences in 1781, Borda proclaimed the superiority of his method against the plurality rule. Rather than considering the candidates all together, he proposed to compare them pairwise, and to select the one who would obtain a majority of votes in all the comparisons. Such a candidate is now called a majority winner or a Condorcet winner $(\mathrm{CW})$. Then, he asserted that the Borda count would always pick a Condorcet winner, whereas the plurality rule would not. Unfortunately, Borda's argument was wrong, and Condorcet [4] provided an example where all the scoring rules, including the Borda count, simultaneously fail to select the majority winner. Table 1.1 
displays this original example. Pierre $\succ$ Paul $\succ$ Jacques represents a preference where Pierre is strictly preferred to Paul, and Paul to Jacques. The second column indicates the number of individuals with such a preference.

Table 1.1

$$
\begin{array}{lr}
\text { Pierre } \succ \text { Paul } \succ \text { Jacques } & 30 \\
\text { Pierre } \succ \text { Jacques } \succ \text { Paul } & 1 \\
\text { Jacques } \succ \text { Pierre } \succ \text { Paul } & 10 \\
\text { Paul } \succ \text { Pierre } \succ \text { Jacques } & 29 \\
\text { Paul } \succ \text { Jacques } \succ \text { Pierre } & 10 \\
\text { Jacques } \succ \text { Paul } \succ \text { Pierre } & 1
\end{array}
$$

In this case, Pierre beats both Paul (41 votes against 40) and Jacques (60 against 21) in pairwise comparisons. However, from a positional point of view, Paul (39 first positions, 31 second places) dominates both Pierre (31 first positions, 39 second places) and Jacques (11 first positions, 11 second places). Irrespective of the scoring vector used, Paul will be the positional winner. We call such a candidate an absolute positional winner (AW). Throughout the rest of this paper, we shall refer to the situations in which the Condorcet and absolute winner are different as Condorcet's profiles.

Modern Social Choice Theory has no provided a solution to this debate. The supporters of Condorcet principle claim that the pairwise comparison methods are more stable when candidates are added or dropped (see, without being exhaustive, Young [27], Saari and Merlin [20]). However, advocates of scoring rules claim that these methods offer higher consistency when voters enter or leave the population (see Young [26], Moulin [18], Merlin and Saari [16]).

The introduction of probability considerations brought a compromise to this debate. Gehrlein and Fishburn [10] define the Condorcet efficiency of a scoring rule as the proportion of voting situations where this positional method selects the Condorcet winner, provided that such a candidate exists. More precisely, they consider populations where each voter selects randomly and independently her preferences according to an uniform probability distribution on the set of linear orderings. This assumption is called the Impartial Culture (IC) condition, as it does not one preference ordering over another. For 
three-candidate elections and the family of scoring vectors $w=(1, \lambda, 0), \lambda \in[0,1]$, they derived an exact formula giving the Condorcet efficiency for large populations as a function of $\lambda$. Under these assumptions, the Borda count maximizes the likelihood of picking the Condorcet winner. Van Newenhizen [25] generalized this result by showing that the Borda rule continues to maximize the Condorcet efficiency among the positional rules for any number of alternatives and a wider class of probability models. Nevertheless, the Borda count fails to satisfy the Condorcet criterion in almost $10 \%$ of the situations in the threecandidate case, and computer calculations indicate that this probability rises as the number of candidates increases (Fishburn and Gehrlein [6]).

The results are improved when we consider more homogeneous societies. A first possibility is to consider the family of Pólya-Eggenberger models (see Berg [1]), where the homogeneity of individual preferences is indexed by a contagion parameter $\alpha \geq 0$. We recover the classical IC assumptions for $\alpha=0$, and the populations becomes more homogeneous as the parameter $\alpha$ increases. As one may guess, these models prove that the Condorcet efficiency increases with $\alpha$. Another possibility is to remove from consideration some types of preferences, assuming for example single-peakedness. In the three-candidate case, Lepelley $[14,15]$ found that this assumption clearly reduces the discrepancies between the majority criterion and the positional rules. Tanguian [24] proposed recently another probability model, assuming the existence of individual cardinal utilities and the existence of their probability distribution. In this particular setup, the probability that the Borda count and Condorcet principle agree converges to one, for any number of candidates as the size of the population increases. Thus, the IC assumption should be considered as a rather extreme case, and the values obtained in this framework could be considered as low evaluations of the Condorcet efficiency measures.

Even if we restrict ourselves to three-candidate elections, the complexity of the computations limits the kind of probabilities we can evaluate and most of the analysis considers the Condorcet efficiency of only one scoring rule at a time. Gehrlein [9] first managed to obtain the joint Condorcet efficiency for the Borda count and the positional rule defined by the scoring vector $(1, \lambda, 0)$. Merlin, Tataru and Valognes [17] developed new techniques and derived an explicit formula for the probability that all the positional rules select simultaneously the Condorcet winner. Gehrlein then recovered some of these results with classical tools [8]. We provide here an analytical formula for the other case, i.e. the probability $P^{\star}$ 
that all the positional rules select the same outcome, but disagree with the majority criterion. Thus, this expression enables us to evaluate the occurrence of Condorcet's profiles, like the one displayed in Table 1.1, in a framework where this phenomenon is relatively frequent. This completes the study of the statistical relationships between the positional rules and the Condorcet principle in the three candidates case inaugurated twenty years ago by Gehrlein and Fishburn. Moreover, from a technical point of view, the techniques developed in this paper and its companion [17] potentially permit us to compute precisely the occurences of any event for three-candidate elections and large electorates under the IC assumption.

The rest of the paper is organized as follows. Section 2 introduces the prerequisites of the model and the process we use to find the desired probability. The results are also displayed and commented on in this section. The techniques we use are quite recent in the literature on the likelihood of voting paradoxes. They have been first introduced by Saari and Tataru [21] and developed in a couple of papers by Tataru and Merlin [23] and Merlin, Tataru and Valognes [17]. Unlike Gehrlein and Fishburn, who base their proofs upon previous results in statistics, we use a geometric argument involving the computation of the volumes of spherical simplexes using Schläfli's formula [5, 13, 22]. This technique and the related computations are presented together in section 3 .

\section{The model and the result}

\subsection{Characterization of the voting situations}

For three-candidate elections, $A=\left\{a_{1}, a_{2}, a_{3}\right\}$. There exist six possible linear orderings on A, and the different preference types are labeled in Table 2.1.

Table 2.1

$$
\begin{array}{llll}
1: & a_{1} \succ a_{2} \succ a_{3} & 4: & a_{3} \succ a_{2} \succ a_{1} \\
2: & a_{1} \succ a_{3} \succ a_{2} & 5: & a_{2} \succ a_{3} \succ a_{1} \\
3: & a_{3} \succ a_{1} \succ a_{2} & 6: & a_{2} \succ a_{1} \succ a_{3}
\end{array}
$$

Let $n$ be the number of individuals, $n_{i}$ the number type $i$ voters and call a vector $\tilde{n}=$ $\left(n_{1}, n_{2}, n_{3}, n_{4}, n_{5}, n_{6}\right)$, representing the distribution of the voters on the different preferences, a voting situation. By definition, $\sum_{i=1}^{6} n_{i}=n$. Without loss of generality, we use the scoring 
vector $w_{s}=(2, s+1,0)$, with $s \in[-1,1]$. We recover the plurality method, the Borda count and the antiplurality rule for $s=-1, s=0$ and $s=1$ respectively.

Saari [19] gave a necessary and sufficient condition for all positional rules to agree. First, a constant scoring rule is defined by the scoring vector $w_{k}=(1,1, \ldots 1,0, \ldots 0)$, $1 \leq k \leq m-1$, with 1's for the first k coordinates and 0's for the remaining ones. Then all the scoring methods give the same results if and only if all the constant rules agree, from $k=1$ to $k=m-1$. In the three-candidate case, this condition says that the plurality and antiplurality rule must select the same winner. Thus, we just have to consider these two scoring rules instead of the continuum defined by the vectors $w_{s}$.

According to Table 2.1, the fact that an alternative, let us say $a_{1}$, has the greatest $w_{s}$-score for a given voting situation is characterized by the following two inequalities:

$$
\begin{aligned}
& (1-s) n_{1}+2 n_{2}+(s+1) n_{3}-(s+1) n_{4}-2 n_{5}+(s-1) n_{6}>0 \\
& 2 n_{1}+(1-s) n_{2}+(s-1) n_{3}-2 n_{4}-(s+1) n_{5}+(s+1) n_{6}>0
\end{aligned}
$$

For the plurality case, $s=-1$, inequalities (2.1) and (2.2) reduce to the inequalities (2.3) and (2.4):

$$
\begin{aligned}
& n_{1}+n_{2}-n_{5}-n_{6}>0 \\
& n_{1}+n_{2}-n_{3}-n_{4}>0
\end{aligned}
$$

In this paper, we investigate the situations where all the scoring rules give the same result, but fail to pick the Condorcet winner. Hence, the Condorcet winner should be $a_{2}$ or $a_{3}$. The fact that $a_{2}$ is a Condorcet winner is given by the inequalities (2.5) and (2.6):

$$
\begin{array}{r}
-n_{1}-n_{2}-n_{3}+n_{4}+n_{5}+n_{6}>0 \\
n_{1}-n_{2}-n_{3}-n_{4}+n_{5}+n_{6}>0
\end{array}
$$

Thus, these six inequalities describe the following event: $a_{1}$ is simultaneously the plurality winner and the $w_{s}$-winner while $a_{2}$ is Condorcet winner. Let $P_{1}(s)$ be the probability that the plurality rule and the $w_{s}$ scoring rule agree while the Condorcet principle select another alternative. Clearly, $P_{1}(s)$ is six times the probability that inequalities (2.1) to (2.6) are fulfilled. When $s=-1$, we recover the probability that the plurality rule fails to select the Condorcet winner; from Gehrlein and Fishburn [10], $P_{1}(-1)=0.22150$. The value of $P_{1}(s)$ is unknown for any other value of $s$. For $s=1$, we shall obtain the probability $P^{\star}$, i.e., the likelihood of Condorcet's profiles. 


\subsection{Probability results under IC assumption}

As mentioned in the introduction, the probability of a voting paradox depends upon the assumptions one may set about the likelihood of the different voting situations. We shall retain throughout this paper the Impartial Culture assumption, which defines the most common model of heterogeneous populations. Thus, the probability that a voter will have the $j^{\text {th }}$ preference type is $\frac{1}{6}$ for three-candidate elections. In turn, $p\left(\tilde{n}=\left(n_{1}, n_{2}, n_{3}, n_{4}, n_{5}, n_{6}\right)\right)$ follows a multinomial distribution; when the size of the population is large enough, the central limit theorem applies and the density distribution tends toward a normal law.

Due to its complexity, the derivation of the exact formulas that gives the value of $P_{1}(s)$ is postponed to section 3 . The results of the computation are presented in Table 2.2. The values for $P_{1}(s)$ are presented in the first column. The second one displays figures from Merlin, Tataru and Valognes [17] about $P_{2}(s)$, the probability that the plurality rule and the $w_{s}$ scoring rule pick together the Condorcet winner. The next one gives the values of $P_{3}(s)$, the probability that the plurality rule and the $w_{s}$-scoring rule agree regardless of any consideration about the majority votes. They are derived from an analytic formula due to Gehrlein and Fishburn [12]. Finally, the last column displays the values of $P_{4}(s)=P_{3}(s)-P_{2}(s)-P_{1}(s)$. It gives the probability that the plurality and the $w_{s}$-rule agree when the Condorcet winner does not exist. 
Table 2.2

\begin{tabular}{||c|c|c|c|c||}
\hline$s$ & $P_{1}(s)$ & $P_{2}(s)$ & $P_{3}(s)$ & $P_{4}(s)$ \\
\hline-1 & 0.22150 & 0.69076 & 1.00000 & 0.08774 \\
-0.8 & 0.18465 & 0.68874 & 0.95684 & 0.08345 \\
-0.6 & 0.14688 & 0.68569 & 0.91031 & 0.07774 \\
-0.4 & 0.11024 & 0.68075 & 0.86102 & 0.07003 \\
-0.2 & 0.07779 & 0.67216 & 0.80994 & 0.05999 \\
0 & 0.05307 & 0.65680 & 0.75834 & 0.05084 \\
0.2 & 0.03762 & 0.63213 & 0.70759 & 0.03784 \\
0.4 & 0.02886 & 0.60053 & 0.65900 & 0.02961 \\
0.6 & 0.02370 & 0.56609 & 0.61359 & 0.02380 \\
0.8 & 0.02039 & 0.53179 & 0.57205 & 0.01987 \\
1 & 0.01808 & 0.49947 & 0.53464 & 0.01709 \\
\hline
\end{tabular}

The most interesting row is the last one, which tells us the probability that all the scoring rules agree $\left(P_{3}(1)\right)$ according to the state of the majority relation: the Condorcet winner is the same outcome $\left(P_{2}(1)\right)$, is different $\left(P_{1}(1)\right)$ or does not exist $\left(P_{4}(1)\right)$. In particular, $P_{1}(1)=P^{\star}$, the likelihood of Condorcet profiles, which turns out to be quite a rare event. Table 2.3 gives the correlation matrix between the existence of an absolute winner and the existence of a Condorcet winner.

Table 2.3

$\begin{array}{cccc} & \exists C W & \nexists C W & \text { Sum } \\ \exists A W & 0.51776 & 0.01709 & 0.53464 \\ \nexists A W & 0.39450 & 0.07065 & 0.46536 \\ \text { Sum } & 0.91226 & 0.08874 & 1.00000\end{array}$

One should first notice that a Condorcet winner is a solution concept far more frequent than an absolute winner. Nevertheless, we should also remark that the Condorcet principle is not unquestioned: there exists profiles where the majority criterion is inconsistent while the positional rules clearly pick up the same winner. 


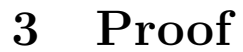

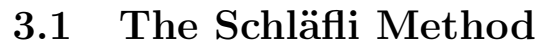

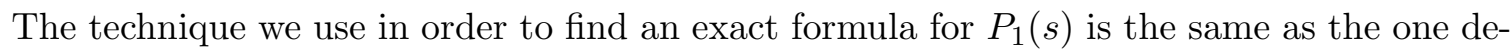
veloped in Merlin, Tataru and Valognes [17]. Let $x_{k}$ be the random variable thas associates to each voter $k$ a vector of the form $(0,0,0,1,0,0)$ with probability $\frac{1}{6}$ of having 1 in each position. Then, the expectation of $x_{k}$ is

$$
E\left(x_{k}\right)=\left(\frac{1}{6}, \frac{1}{6}, \frac{1}{6}, \frac{1}{6}, \frac{1}{6}, \frac{1}{6}\right)
$$

and the covariance matrix is a diagonal $6 \times 6$ matrix with the common entry $\sigma$ given by

$$
\sigma^{2}=E\left(x_{k}^{2}\right)-E\left(x_{k}\right)^{2} .
$$

Let

$$
m^{T}=\left(m_{1}, m_{2}, \ldots m_{6}\right)^{T}=\frac{1}{\sigma \sqrt{n}}\left[\left(\begin{array}{c}
n_{1} \\
\vdots \\
n_{6}
\end{array}\right)-\left(\begin{array}{c}
\frac{n}{6} \\
\vdots \\
\frac{n}{6}
\end{array}\right)\right]
$$

The Central Limit Theorem in $\mathbb{R}^{5}$ implies the following convergence in measurr:

$$
\mu\left[m^{T}\right] \mapsto \frac{1}{(\sqrt{2} \pi)^{5}} e^{\frac{-|t|^{2}}{2}} \lambda
$$

as $n \rightarrow \infty$ where $t=\left(t_{1}, t_{2}, \ldots, t_{6}\right) \in \mathbb{R}^{6},|t|^{2}=t_{1}^{2}+\ldots+t_{6}^{2}$ and $\lambda$ is Lebesgue measure on the five-dimensional hyperplane $t_{1}+\ldots+t_{6}=0$. Note that since $m^{T}$ has the measure supported on the hyperplane $m_{1}+\ldots+m_{6}=0$, the limit of $m^{T}$ as $n \rightarrow \infty$ is also a measure supported on $t_{1}+\ldots+t_{6}=0$. In order to compute $P_{1}(s)$, we shall evaluate the probablity that a voting situation fulfills the set of inequalities (2.1) to (2.6). By subtracting or dividing the number of voters of each type by the same constant, the quantities change, but the comparisons between them are unchanged, therefore, one can easily claim that $\tilde{n}^{T}$ satisfies conditions (2.1) to (2.6) if and only if $m^{T}$ satisfies these inequalities. The Central Limit Theorem yields

$$
P\left(m^{T} \quad \text { satisfies } \quad(2.1) \quad \text { to } \quad(2.6)\right) \mapsto \frac{1}{(\sqrt{2} \pi)^{5}} \int_{C_{1}} e^{\frac{-|t|^{2}}{2}} d \lambda
$$


where $C_{1}=\left\{t \in \mathbb{R}^{6}, t\right.$ satisfies (2.1) to (2.6) and $\left.\sum_{i=1}^{6}\left(t_{i}\right)=0\right\}$. Because conditions (2.1) to (2.6) are homogeneous, the domain $C_{1}$ of the integration is a cone. Also the measure

$$
\bar{\mu} \equiv \frac{1}{(\sqrt{2} \pi)^{5}} e^{\frac{-|t|^{2}}{2}} \lambda
$$

is absolutely continuous and radially symmetric. Hence computing

$$
\frac{1}{(\sqrt{2} \pi)^{5}} \int_{C_{1}} e^{\frac{-|t|^{2}}{2}} d \lambda
$$

reduces to finding the measure $\bar{\mu}$ of the cone $C_{1}$, when the measure is invariant to rotations. The measure $\bar{\mu}$ of such a cone is proportional to the euclidian measure of the cone, that is the measure on the sphere.

As the cone $C_{1}$ lies on the five-dimensional subspace $\sum_{i=1}^{6} t_{i}=0$, its intersection with the five-dimensional hypersphere is a four-dimensional spherical simplex. Schläfli's formula $[5,13,22]$ gives the differential volume of a $p$-dimensional spherical simplex as a function of the volume of the intersections between any two faces $S_{j}, S_{k}$ and the dihedral angle between these faces.

$$
\operatorname{dvol}_{p}(C)=\frac{1}{(p-1)} \sum_{1 \leq j<k \leq n} \operatorname{vol}_{p-2}\left(S_{j} \cap S_{k}\right) d \alpha_{j k} ; \quad \operatorname{vol}_{0}=1
$$

where $\alpha_{j k}$ is the dihedral angle formed by the facets $S_{j}, S_{k}$ of C. To apply this formula to our problem, let us denote by $S_{j}$ the facet defined by the equation (2.j), and $S_{7}$ the equation $\sum_{i=1}^{6} t_{i}=0$. We shall now evaluate the different components in formula (3.1).

\subsection{Computation of the dihedral angles}

For each equation (2.1) to $(2.6)$, let $\vec{V}_{j}$ be a normal vector to the hyperplane $S_{j}$ :

$$
\begin{array}{lll}
\overrightarrow{V_{1}}=(1-s, 2, s+1,-s-1,-2, s-1), & \left\|\overrightarrow{V_{1}}\right\|=2 \sqrt{s^{2}+3} \\
\overrightarrow{V_{2}}=(2,1-s, s-1,-2,-s-1, s+1), & \left\|\overrightarrow{V_{2}}\right\|=2 \sqrt{s^{2}+3} \\
\overrightarrow{V_{3}}=(1,1,0,0,-1,-1), & \left\|\overrightarrow{V_{3}}\right\|=2 \\
\overrightarrow{V_{4}}=(1,1,-1,-1,0,0), & \left\|\vec{V}_{4}\right\|=2 \\
\overrightarrow{V_{5}}=(-1,-1,-1,1,1,1), & \left\|\overrightarrow{V_{5}}\right\|=\sqrt{6} \\
\overrightarrow{V_{6}}=(1,-1,-1,-1,1,1), & \left\|\overrightarrow{V_{6}}\right\|=\sqrt{6}
\end{array}
$$

$\vec{V}_{j}$ and $\vec{V}_{k}$ are respectively normal to $S_{j}$ and $S_{k}$. Hence:

$$
\alpha_{j k}=\arccos \left(\frac{-\vec{V}_{j} \cdot \vec{V}_{k}}{\left\|\vec{V}_{j}\right\| \cdot\left\|\vec{V}_{k}\right\|}\right)
$$


As we shall derive the dihedral angles, we are only interested in the angles which depend on $s$. There are 8 of them:

$$
\begin{aligned}
& \alpha_{15}=\arccos \left(\frac{4}{\sqrt{6\left(s^{2}+3\right)}}\right) \\
& \alpha_{26}=\arccos \left(\frac{-2}{\sqrt{6\left(s^{2}+3\right)}}\right) \\
& \alpha_{16}=\alpha_{25}=\arccos \left(\frac{2}{\sqrt{6\left(s^{2}+3\right)}}\right) \\
& \alpha_{13}=\alpha_{24}=\arccos \left(\frac{s-3}{2 \sqrt{3+s^{2}}}\right) \\
& \alpha_{14}=\alpha_{23}=\arccos \left(\frac{s-3}{4 \sqrt{3+s^{2}}}\right)
\end{aligned}
$$

Hence:

$$
\begin{aligned}
& d \alpha_{15}=\frac{-4 s}{\left(s^{2}+3\right) \sqrt{6 s^{2}+18}} d s \\
& d \alpha_{26}=\frac{2 s}{\left(s^{2}+3\right) \sqrt{6 s^{2}+18}} d s \\
& d \alpha_{16}=d \alpha_{25}=\frac{-2 s}{\left(s^{2}+3\right) \sqrt{\left.6 s^{2}+18\right)}} d s \\
& d \alpha_{13}=d \alpha_{24}=\frac{3+3 s}{2\left(3+s^{2}\right) \sqrt{3+s^{2}}} d s \\
& d \alpha_{14}=d \alpha_{23}=\frac{3+3 s}{4\left(3+s^{2}\right) \sqrt{3+s^{2}}} d s
\end{aligned}
$$

\subsection{Finding the Vertices}

The dihedral volumes $S_{j} \cap S_{k}$ are two-dimensional spherical simplexes, and we shall first find their vertices. A direction in $\mathbb{R}^{5}$ is given by solving a system of four linear equations. Thus, we shall find the coordinates of a vertex, $P_{1234}$ for example, by solving the following system:

$$
\left\{\begin{array}{l}
S_{1}=0 \\
S_{2}=0 \\
S_{3}=0 \\
S_{4}=0 \\
S_{5}>0 \\
S_{6}>0 \\
S_{7}=0
\end{array}\right.
$$

Among the 15 systems of equations, only eight of them will provide an adequate solutions. Moreover, we shall distinguish two cases: $s \leq 0$ and $s \geq 0$. 
case 1: $s \leq 0$

$$
\begin{aligned}
& P_{1256}=(0,1+s,-1-s, 0,1-s, s-1) \\
& P_{1356}=(-1,2,-1,-1,2,-1) \\
& P_{1245}=(-2 s+2,3 s-1,-s-1,2 s+2,-3 s-1, s-1) \\
& P_{1246}=(2 s+2,-s-1,3 s-1,-2 s+2, s-1,-3 s-1) \\
& P_{2456}=(2,-1,-1,2,-1,-1) \\
& P_{1345}=(1,-1,0,0,-1,1) \\
& P_{1346}=(0,0,-1,1,-2,2) \\
& P_{3456}=(0,0,0,0,-1,1)
\end{aligned}
$$

case $2: s \geq 0$

$$
\begin{aligned}
& P_{1256}=(0,1+s,-1-s, 0,1-s, s-1) \\
& P_{1235}=(s-1,2,-s-1,-s-1,2, s-1) \\
& P_{1236}=(-s-1,2+2 s,-3 s-1, s-1,2-2 s, 3 s-1) \\
& P_{2356}=(-1-s, 2+2 s,-s-1,-s-1,2-s, 2 s-1) \\
& P_{1456}=(2+2 s,-s-1,-s-1,2 s+2,-7 s-1,5 s-1) \\
& P_{1345}=(1,-1,0,0,-1,1) \\
& P_{1346}=(0,0,-1,1,-2,2) \\
& P_{3456}=(0,0,0,0,-1,1)
\end{aligned}
$$

Also notice that the faces defined by the inequalities (2.2) and (2.3) do not intersect in the first case. The same situation holds with the faces defined by the inequalities (2.2) and (2.4) for $s \geq 0$. According to each case, the spherical simplex $S_{j} \cap S_{k}$ could possess 3 or 4 vertices, or simply does not exist. This data is summarized in Table 3.1. $P_{a b c d}$ is a vertex 
for $S_{j} \cap S_{k}$ if $j$ and $k$ appear as indices.

Table 3.1

\begin{tabular}{||c|l|l||}
\hline Volumes & Vertices, $s \leq 0$ & Vertices, $s \geq 0$ \\
\hline$S_{1} \cap S_{3}$ & $P_{1356}, P_{1345}, P_{1346}$ & $P_{1235}, P_{1236}, P_{1345}, P_{1346}$ \\
$S_{1} \cap S_{4}$ & $P_{1245}, P_{1246}, P_{1345}, P_{1346}$ & $P_{1456}, P_{1345}, P_{1346}$ \\
$S_{1} \cap S_{5}$ & $P_{1256}, P_{1356}, P_{1245}, P_{1345}$ & $P_{1256}, P_{1235}, P_{1456}, P_{1345}$ \\
$S_{1} \cap S_{6}$ & $P_{1256}, P_{1356}, P_{1246}, P_{1346}$ & $P_{1256}, P_{1236}, P_{1456}, P_{1346}$ \\
$S_{2} \cap S_{3}$ & $\emptyset$ & $P_{1235}, P_{1236}, P_{2356}, P_{1346}$ \\
$S_{2} \bigcap S_{4}$ & $P_{1245}, P_{1246}, P_{2456}$ & $\emptyset$ \\
$S_{2} \cap S_{5}$ & $P_{1256}, P_{1245}, P_{2456}$ & $P_{1256}, P_{1356}, P_{2356}$ \\
$S_{2} \cap S_{6}$ & $P_{1256}, P_{1246}, P_{2456}$ & $P_{1256}, P_{1236}, P_{2356}$ \\
\hline
\end{tabular}

\subsection{Differential volumes}

The differential volumes reduce to surfaces of two-dimensional simplexes on the sphere. Thus, we can apply the Gauss-Bonnet theorem: the surface equals to the sum of the angles on the surface minus $\pi$. We describe the details of the computations for the differential volume $S_{2} \bigcap S_{4}$ in case $s \leq 0$. The other cases can be worked in a similar fashion.

Let $\beta_{15}, \beta_{16}$ and $\beta_{56}$ be the angles on the surface of the triangle, defined by the vertices $P_{1245}, P_{1246}$ and $P_{2456} ; \delta_{1}, \delta_{5}$ and $\delta_{6}$ are respectively the angles $P_{1245}, \widehat{P} P_{1246}, P_{1245}, P_{2456}$ and $P_{1246}, P_{2456}$. Hence:

$$
\begin{aligned}
& \cos \left(\beta_{15}\right)=\frac{\cos \left(\delta_{6}\right)-\cos \left(\delta_{1}\right) \cos \left(\delta_{5}\right)}{\sin \left(\delta_{1}\right) \sin \left(\delta_{5}\right)} \\
& \cos \left(\beta_{16}\right)=\frac{\cos \left(\delta_{5}\right)-\cos \left(\delta_{1}\right) \cos \left(\delta_{6}\right)}{\sin \left(\delta_{1}\right) \sin \left(\delta_{6}\right)} \\
& \cos \left(\beta_{56}\right)=\frac{\cos \left(\delta_{1}\right)-\cos \left(\delta_{5}\right) \cos \left(\delta_{6}\right)}{\sin \left(\delta_{5}\right) \sin \left(\delta_{6}\right)}
\end{aligned}
$$

In our case:

$$
\begin{gathered}
\cos \left(\delta_{1}\right)=\frac{3-5 s^{2}}{3+7 s^{2}} \\
\cos \left(\delta_{5}\right)=\cos \left(\delta_{6}\right)=\frac{\sqrt{3}}{\sqrt{3+7 s^{2}}}
\end{gathered}
$$


Hence, by (3.2) to (3.6)

$$
\begin{aligned}
\operatorname{Vol}_{p-2}\left(S_{2} \bigcap S_{4}\right) & =\beta_{15}+\beta_{16}+\beta_{56}-\pi \\
& =+\arccos \left(\frac{5}{7}\right)+2 \arccos \left(\frac{3 \sqrt{\frac{2}{7}}}{\sqrt{3+s^{2}}}\right)
\end{aligned}
$$

The same reasoning enables us to find exact formulas for:

- $I_{1}(s)=\sum_{j=1,2 k=3456} \operatorname{Vol}_{p-2}\left(S_{j} \bigcap S_{k}\right) d \alpha_{j k}, s \leq 0$.

- $I_{2}(s)=\sum_{j=1,2 k=3456} \operatorname{Vol}_{p-2}\left(S_{j} \cap S_{k}\right) d \alpha_{j k}, s \geq 0$.

Due to the fact that the event we analyze involves two different alternatives, there is no symmetry among the dihedral volumes; contrary to computations in Merlin, Tataru and Valognes [17], there is no simplification and we need to treat separately the fourteen cases. After simplifications, formulas for $I_{1}(s)$ and $I_{2}(s)$ are given by the expressions (3.9) and (3.10):

$$
\begin{aligned}
I_{1}(s)= & \left.+\frac{4 s\left(\arccos \left(\frac{s}{\sqrt{1+4 s^{2}}}\right)-\arccos \left(\frac{+1+s}{\sqrt{13+2 s+37 s^{2}}}\right)+\arccos \left(\frac{(-3+s) \sqrt{3+9 s^{2}}}{\sqrt{\left(3+s^{2}\right)\left(13+2 s+37 s^{2}\right)}}\right)-\arccos \left(\sqrt{\frac{3+9 s^{2}}{3+13 s^{2}+4 s^{4}}}\right)\right)}{\left(3+s^{2}\right) \sqrt{2+6 s^{2}}}\right) \\
& -\frac{2 s\left(2 \arccos \left(\frac{2 s}{\sqrt{7+7 s^{2}}}\right)-2 \arccos \left(\frac{2 \sqrt{\frac{3}{7}} s}{\sqrt{3+10 s^{2}+3 s^{4}}}\right)-\arccos \left(\frac{3\left(-1+s^{2}\right)}{\sqrt{\left(3+12 s^{2}\right)\left(3+10 s^{2}+3 s^{4}\right)}}\right)\right)}{\left(3+s^{2}\right) \sqrt{14+6 s^{2}}} \\
& +\frac{2 s\left(\arccos \left(\frac{5 s}{\sqrt{7} \sqrt{1+4 s^{2}}}\right)-\arccos \left(\frac{7+13 s}{\sqrt{7} \sqrt{37+26 s+37 s^{2}}}\right)-\arccos \left(\frac{\sqrt{3}(1-s)\left(3-2 s+3 s^{2}\right)}{\sqrt{\left(37+26 s+37 s^{2}\right)\left(3+10 s^{2}+3 s^{4}\right)}}\right)\right)}{\left(3+s^{2}\right) \sqrt{14+6 s^{2}}} \\
& -\frac{\sqrt{3}\left(\frac{\pi}{2}+\arccos \left(\frac{1}{\sqrt{7}}\right)-\arccos \left(\frac{3}{\sqrt{14}}\right)-\arccos \left(\frac{5}{7}\right)+2 \arccos \left(\frac{3 \sqrt{\frac{2}{7}}}{\sqrt{3+s^{2}}}\right)\right)}{3+s^{2}} \\
& -\frac{\sqrt{3}(1+s)\left(-\arccos \left(\frac{6 s-2 s^{2}}{\sqrt{\left(6+2 s^{2}\right)\left(13+2 s+37 s^{2}\right)}}\right)+\arccos \left(\frac{12+6 s+2 s^{2}}{\sqrt{\left(6+2 s^{2}\right)\left(37+26 s+37 s^{2}\right)}}\right)\right)}{\left(3+s^{2}\right) \sqrt{13+2 s+5 s^{2}}} \\
& -\frac{\sqrt{3}(1+s)\left(\arccos \left(\frac{8 s}{\sqrt{26+4 s+74 s^{2}}}\right)+\arccos \left(\frac{3+7 s}{\sqrt{74+52 s+74 s^{2}}}\right)\right)}{\left(3+s^{2}\right) \sqrt{13+2 s+5 s^{2}}}
\end{aligned}
$$




$$
\begin{aligned}
& I_{2}(s)=\frac{4 s\left(\frac{3 \pi}{2}-\arccos \left(\frac{1+s}{\sqrt{13+2 s+37 s^{2}}}\right)+\arccos \left(\frac{-3+s-8 s^{2}}{\sqrt{\left(1+4 s^{2}\right)\left(13+2 s+37 s^{2}\right)}}\right)-\arccos \left(\sqrt{\frac{3+9 s^{2}}{3+13 s^{2}+4 s^{4}}}\right)\right)}{\left(3+s^{2}\right) \sqrt{2+6 s^{2}}} \\
& -2 s\left(\arccos \left(\frac{3+2 s+5 s^{2}}{\sqrt{\left(1+s^{2}\right)\left(37+26 s+37 s^{2}\right)}}\right)+\arccos \left(\frac{\sqrt{3}(-1+s)\left(3-2 s+3 s^{2}\right)}{\sqrt{\left(37+26 s+37 s^{2}\right)\left(3+10 s^{2}+3 s^{4}\right)}}\right)\right) \\
& \left(3+s^{2}\right) \sqrt{14+6 s^{2}} \\
& +\frac{2 s\left(-\arccos \left(\frac{7+13 s}{\sqrt{7} \sqrt{37+26 s+37 s^{2}}}\right)+\arccos \left(\frac{(1+s)(-3+8 s)}{\sqrt{1+4 s^{2}} \sqrt{37+26 s+37 s^{2}}}\right)+\arccos \left(\frac{3\left(-1+s^{2}\right)}{\sqrt{\left(3+12 s^{2}\right)\left(3+10 s^{2}+3 s^{4}\right)}}\right)\right)}{\left(3+s^{2}\right) \sqrt{14+6 s^{2}}} \\
& +\frac{2 s\left(-\arccos \left(\frac{2 \sqrt{\frac{3}{7}} s}{\sqrt{3+10 s^{2}+3 s^{4}}}\right)+\arccos \left(\frac{(-3+9 s) \sqrt{3+s^{2}}}{\sqrt{3+9 s^{2}} \sqrt{37+2 s+13 s^{2}}}\right)+\arccos \left(\frac{3-2 s+s^{2}}{\sqrt{\left(1+s^{2}\right)\left(37+2 s+13 s^{2}\right)}}\right)\right)}{\left(3+s^{2}\right) \sqrt{14+6 s^{2}}} \\
& -\frac{\sqrt{3}\left(-\arccos \left(\frac{3}{\sqrt{14}}\right)+\arccos \left(\frac{-6 \sqrt{5}}{\sqrt{210+70 s^{2}}}\right)\right)}{3+s^{2}}
\end{aligned}
$$

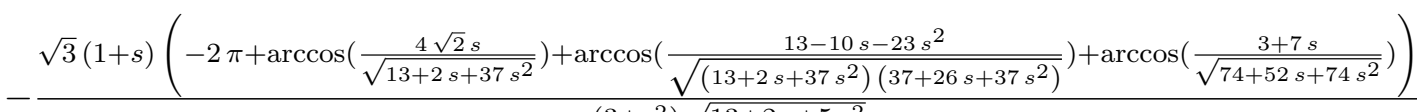

$$
\begin{aligned}
& \left(3+s^{2}\right) \sqrt{13+2 s+5 s^{2}} \\
& -\frac{\sqrt{3}(1+s)\left(\arccos \left(\sqrt{\frac{24+8 s^{2}}{37+2 s+13 s^{2}}}\right)+\arccos \left(\frac{12+6 s+2 s^{2}}{\sqrt{\left(6+2 s^{2}\right)\left(37+26 s+37 s^{2}\right)}}\right)+\arccos \left(\frac{-11-10 s+s^{2}}{\sqrt{\left(37+2 s+13 s^{2}\right)\left(37+26 s+37 s^{2}\right)}}\right)\right)}{\left(3+s^{2}\right) \sqrt{13+2 s+5 s^{2}}}
\end{aligned}
$$

Between -1 and 0 , the differential volume is $\frac{I_{1}}{3}$ as $p=4$ in the Schläfli's formula. We have to multiply this number by 6 , and to divide it by the surface of the hypersphere in $\mathbb{R}^{5}$, $\omega_{5}=\frac{8 \pi^{2}}{3}$. Thus, the probability that the plurality rule and the $w_{s}$-rule, $s \leq 0$, agree while the Condorcet criterion selects another alternative is given by equation (3.11).

$$
P_{1}(s)=0.22150+\frac{3}{4 \pi^{2}} \int_{-1}^{s} I_{1}(u) d u
$$

Recall that value 0.22150 comes from Gehrlein and Fishburn [10]. The same reasoning applies for the case $s \in[0,1]$ and the formula of $P_{1}(s)$ is then given by equation (3.12).

$$
P_{1}(s)=P_{1}(0)+\frac{3}{4 \pi^{2}} \int_{0}^{s} I_{2}(u) d u
$$

The numerical values of the Table 2.2 are computed by performing the integration in the above formulas. 


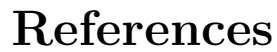

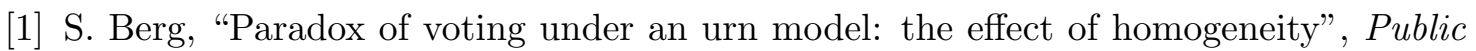

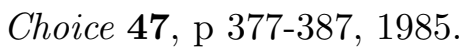

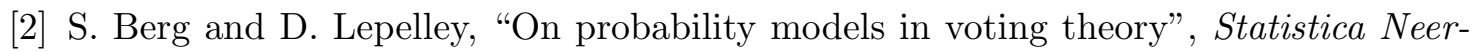

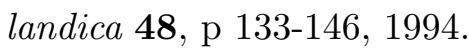

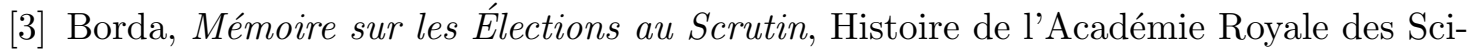

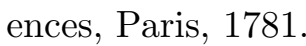

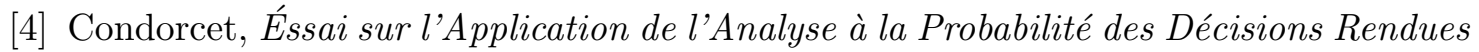

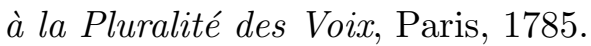

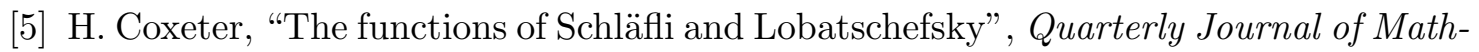

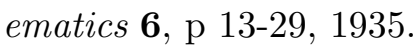

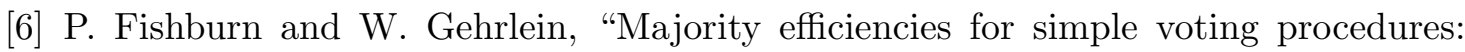

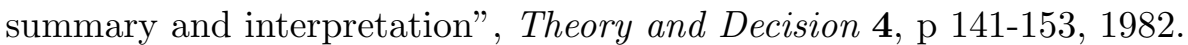

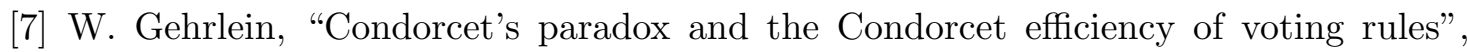

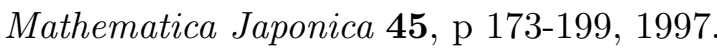

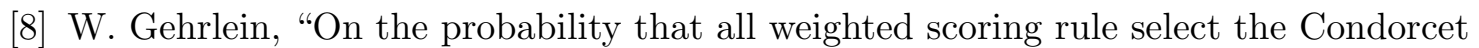

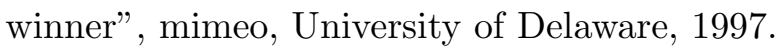

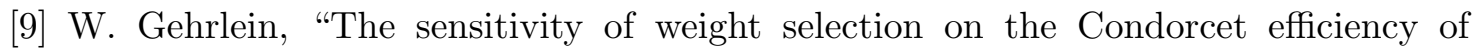

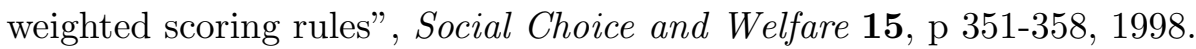

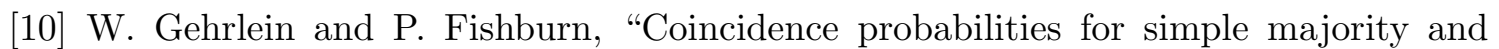

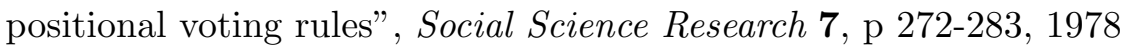

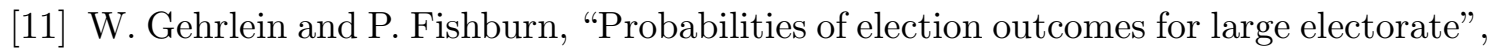
Journal of Economic Theory 19, p 38-49, 1978.

[12] W. Gehrlein and P. Fishburn, "Scoring rule sensitivity to weight selection", Public Choice 40, p 249-261, 1983.

[13] R. Kellerhals, "On the volume of hyperbolic polyhedra", Mathematische Annalen 285, p 541-569, 1989. 
[14] D. Lepelley, "Condorcet efficiency of positional voting rules with single-peaked preferences", Economic Design 1, p 289-299, 1995.

[15] D. Lepelley, "Constant scoring rules, Condorcet criteria and single-peaked preferences", Economic Theory 7, p 491-500, 1996.

[16] V. Merlin and D. Saari, "The Copeland method II: manipulations, monotonicity and paradoxes", Journal of Economic Theory 72, p 148-172, 1997.

[17] V. Merlin, M. Tataru and F. Valognes, "On the probability that all the rules select the same winner", Journal of Mathematical Economics. To appear.

[18] H. Moulin, "Condorcet's principle implies the no show paradox", Journal of Economic Theory 45 p 45-53, 1988.

[19] D. Saari, "Millions of election rankings from a single profile", Social Choice and Welfare $\mathbf{9}, \mathrm{p}$ 277-306, 1992.

[20] D. Saari and V. Merlin, "The Copeland method I: relationships and the dictionary", Economic Theory 8, p 51-76, 1996.

[21] D. Saari and M. Tataru, "The likelihood of dubious election outcomes", Economic Theory. To appear.

[22] L. Schläfli, Theorie der Vielfachen Kontinuität, Gesmmelte Mathematische Abhandlungen 1, Birkhäuser, Basel, 1950.

[23] M. Tataru and V. Merlin, "On the relationship of the Condorcet winner and positional voting rules", Mathematical Social Sciences 34, pp 81-90, 1997.

[24] A. Tanguian, "Solution to Condorcet's paradox", paper presented at the 3rd International Meeting of the Society for Social Choice and Welfare, Maastricht, 1996.

[25] J. van Newenhizen, "The Borda method is the most likely to respect the Condorcet principle", Economic Theory 2, p 69-83, 1992.

[26] P. Young, "Social Choice Scoring Functions", SIAM Journal of Applied Mathematics 28, p 824-838, 1975. 
[27] P. Young, "Condorcet's theory of voting", American Political Science Review 82 p 1231-1244, 1988. 